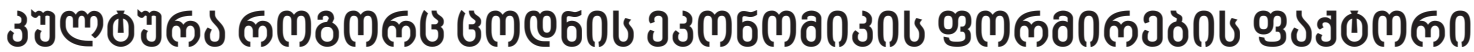

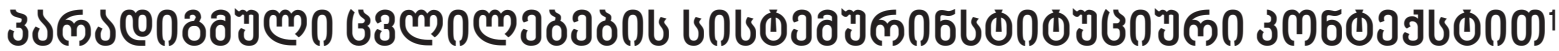

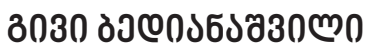

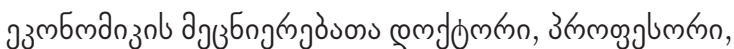 \\ эз

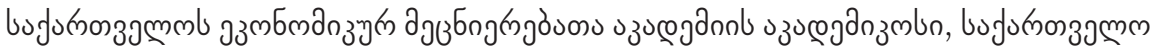 \\ g.bedianashvili@eu.edu.ge
}

https://doi.org/10.35945/gb.2018.06.007

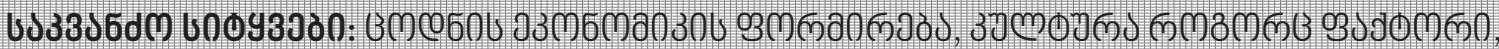

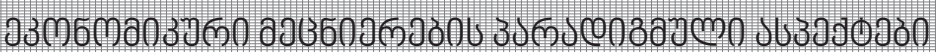

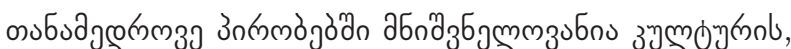

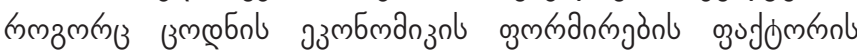

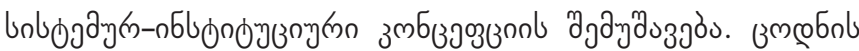

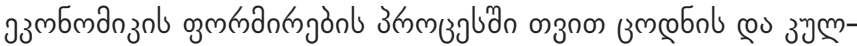

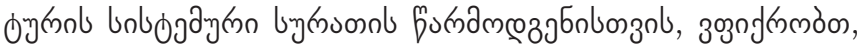

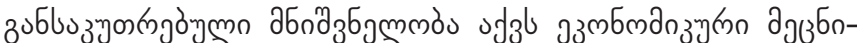

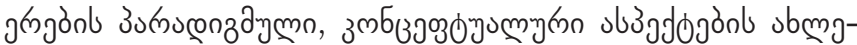

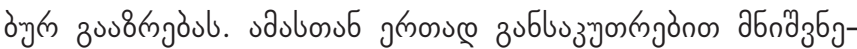

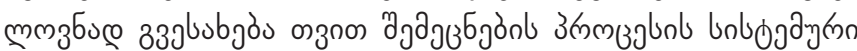

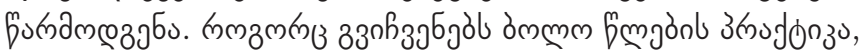

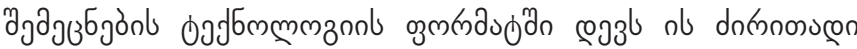

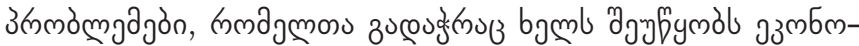

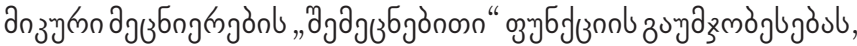

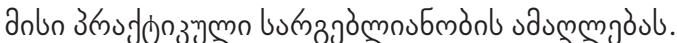

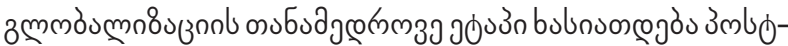

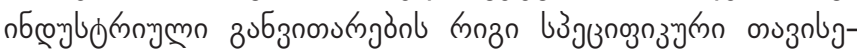

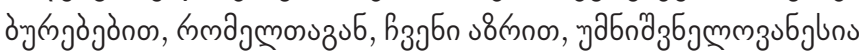

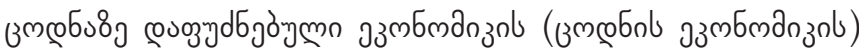

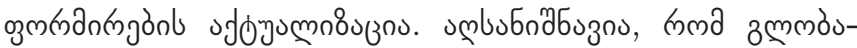

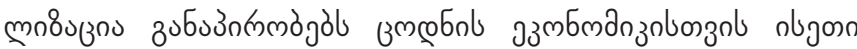

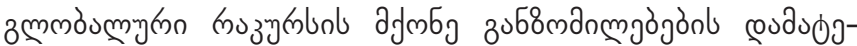

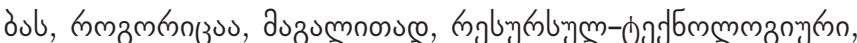

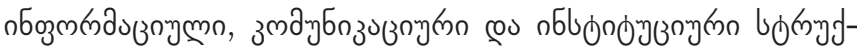

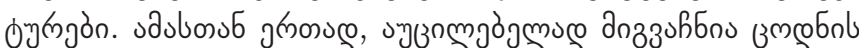

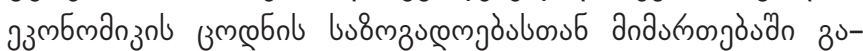

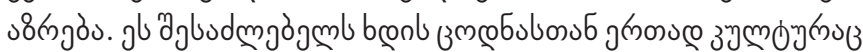

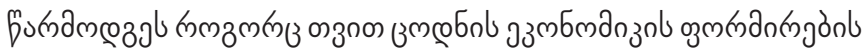
oुsdomm

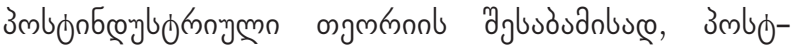

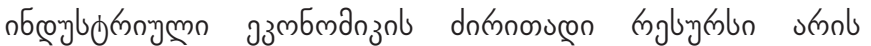

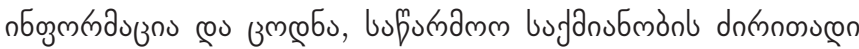

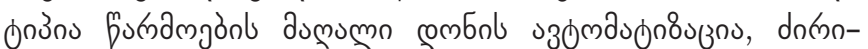

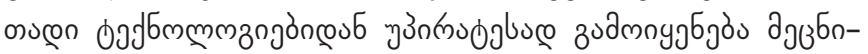

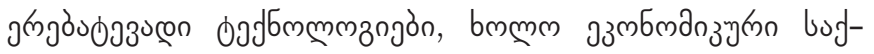

1 ззмлзก

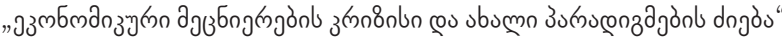

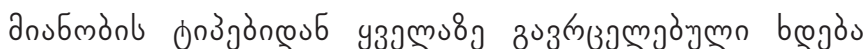

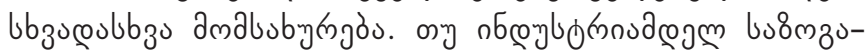

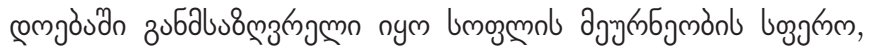

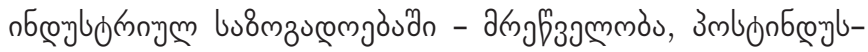

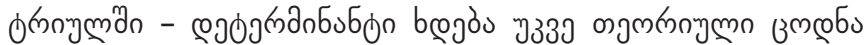

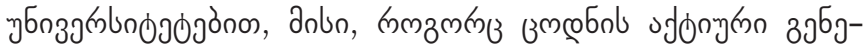

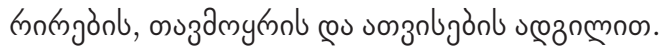

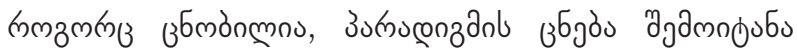

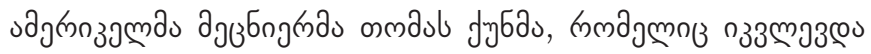

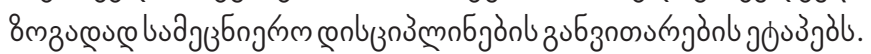

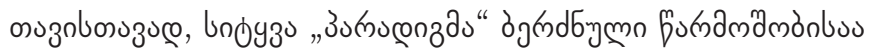

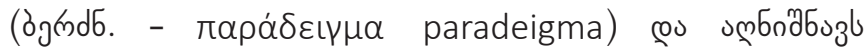

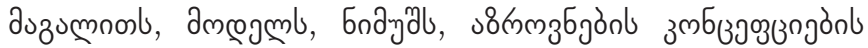

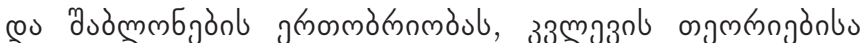

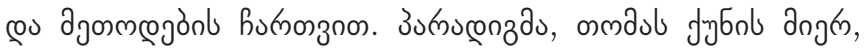

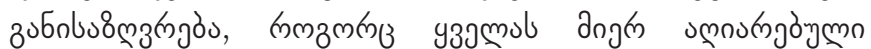

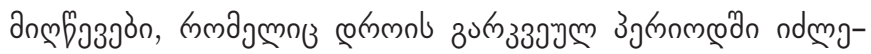

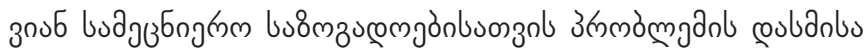

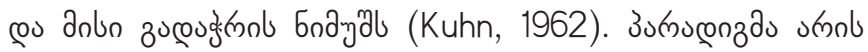

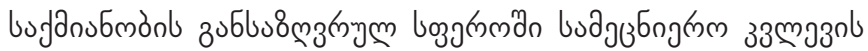

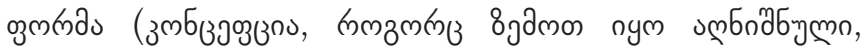

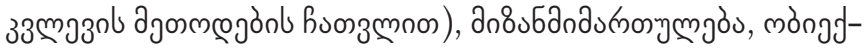

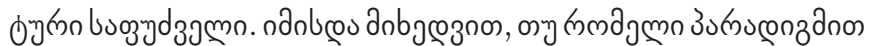

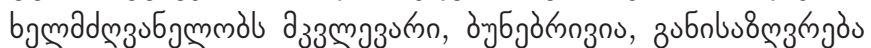

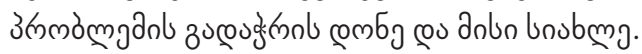

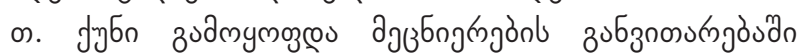

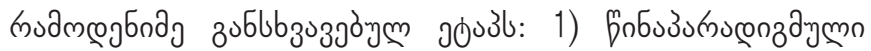

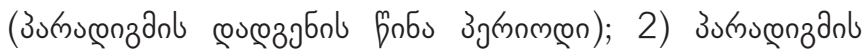

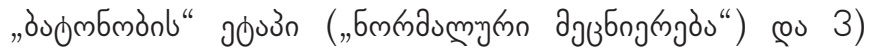

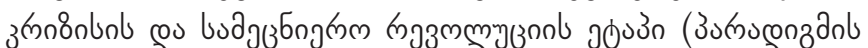

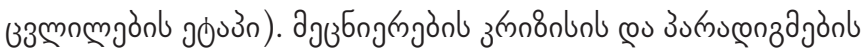

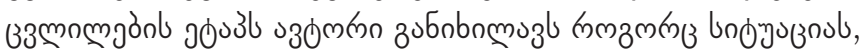

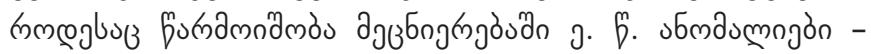

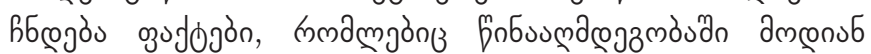

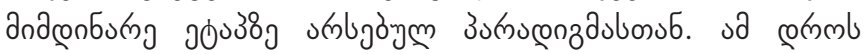

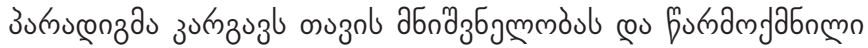

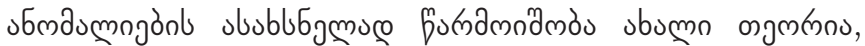




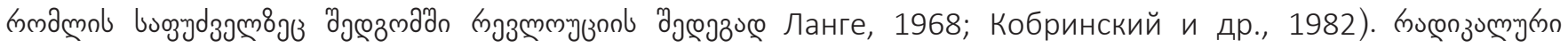

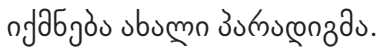

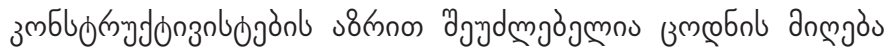

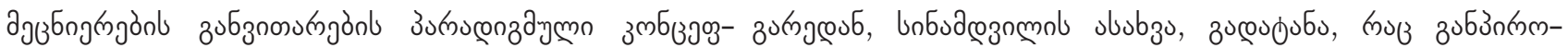

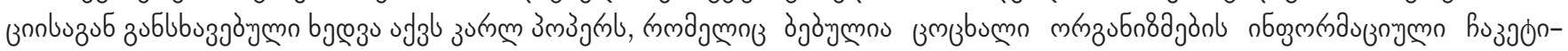

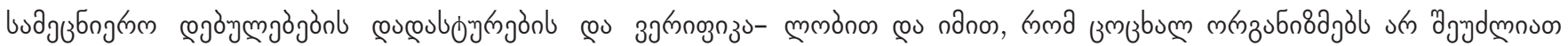

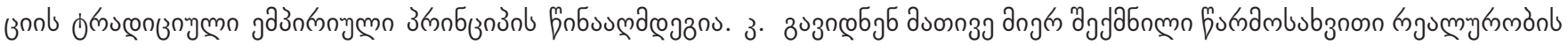

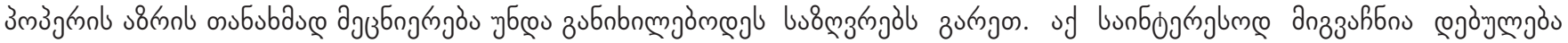

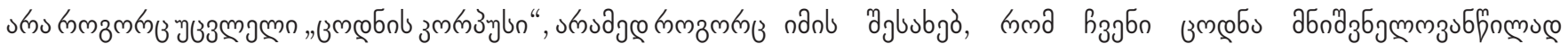

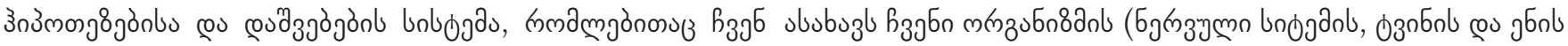

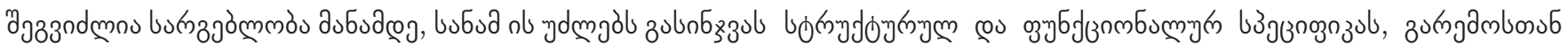
cos żampipajobl (Popper, 1980).

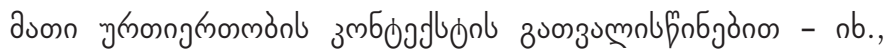

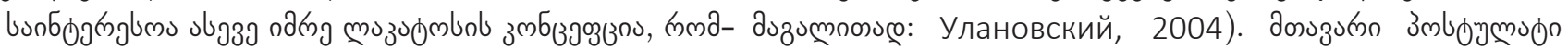

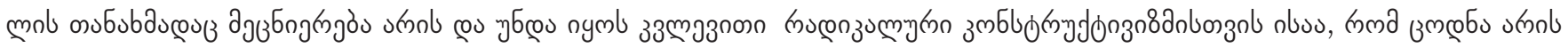

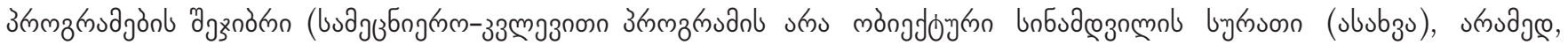

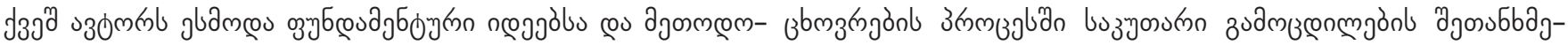

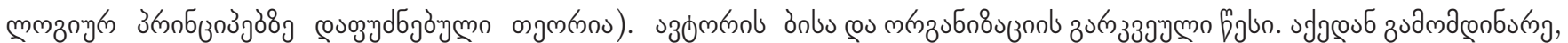

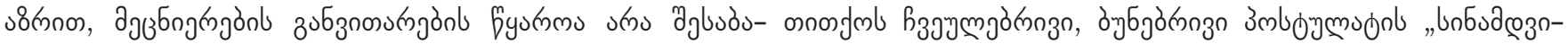

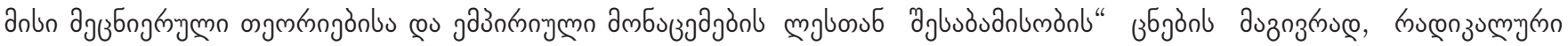

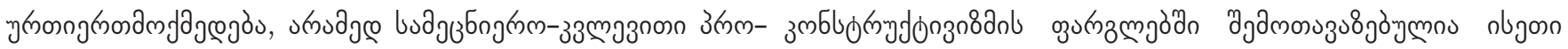

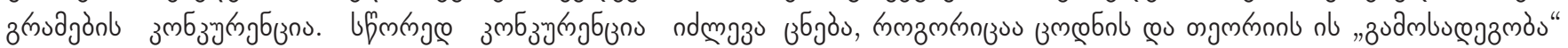

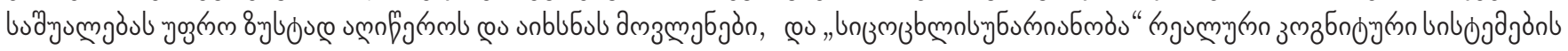

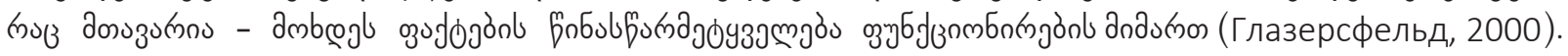

(Lakatos, 1970).

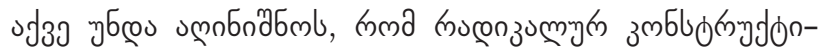

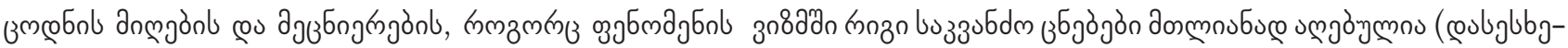

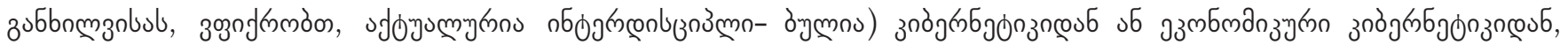

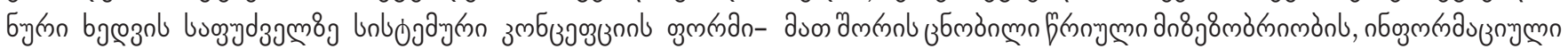

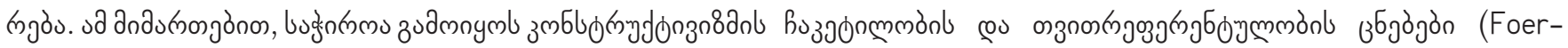

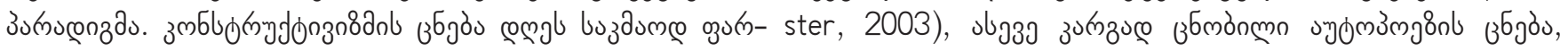

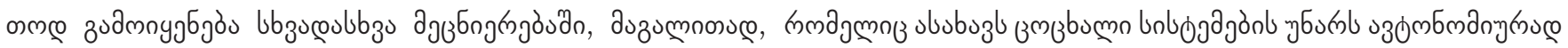

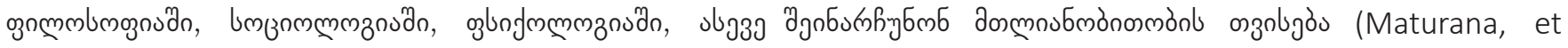

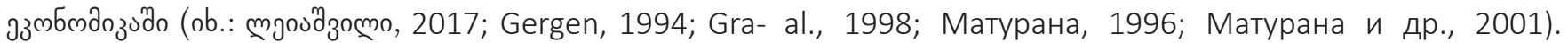

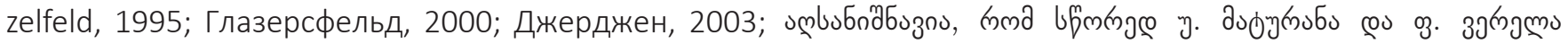

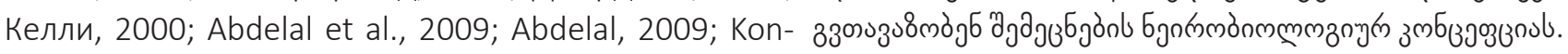

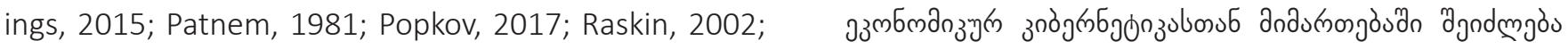

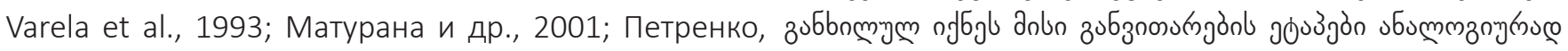

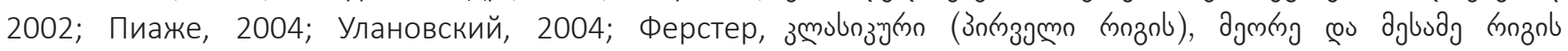

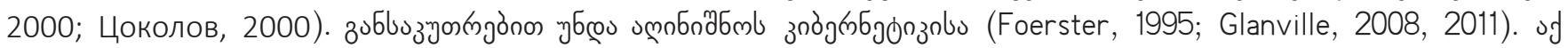

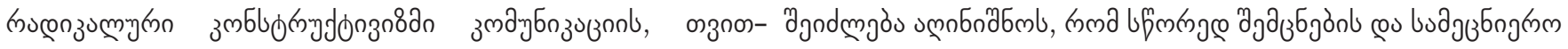

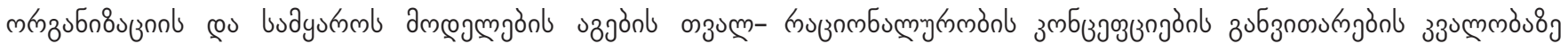

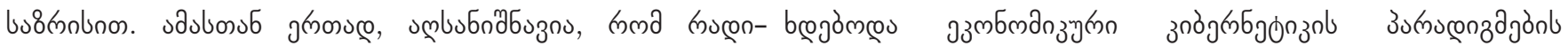

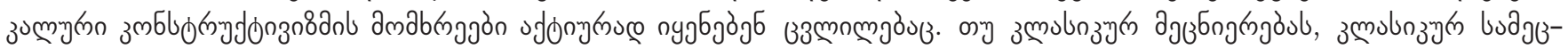

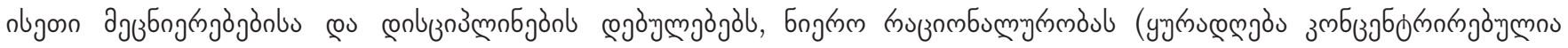

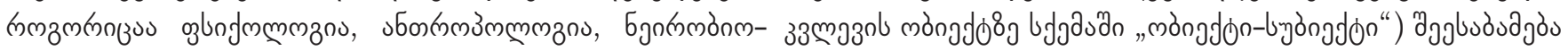

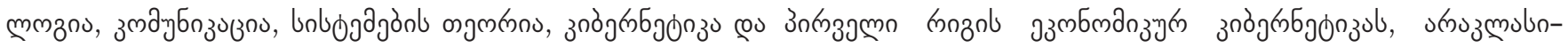

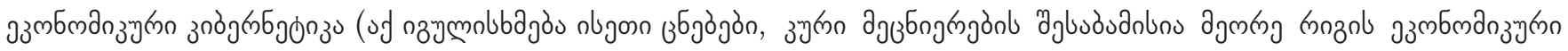

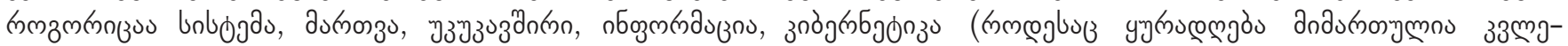

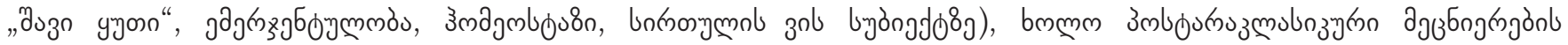

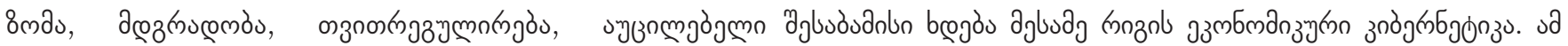

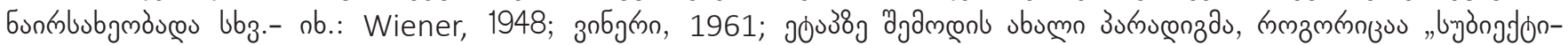




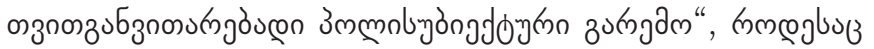

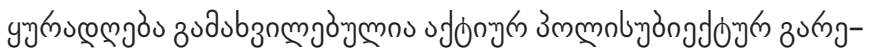

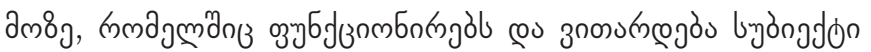
(оь., дјзимпполю: Лепский, 2015).

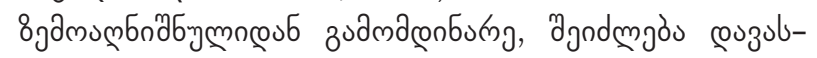

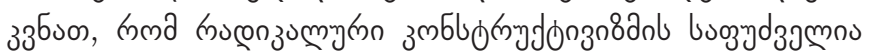

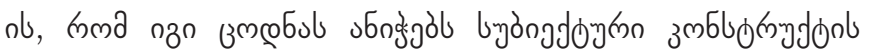

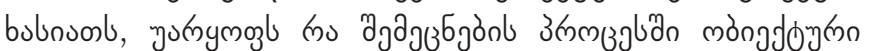

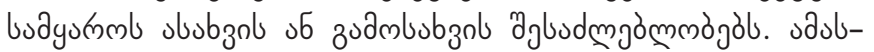

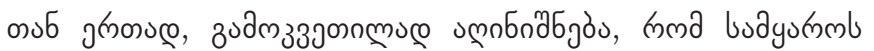

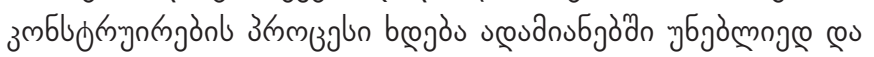

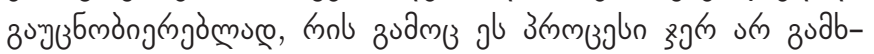

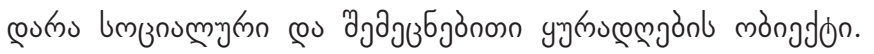

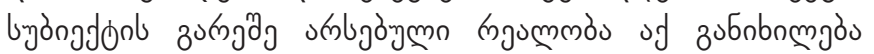

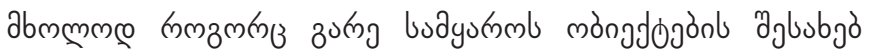

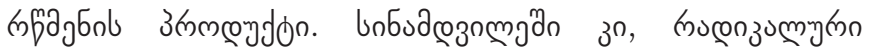

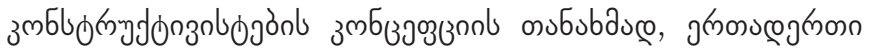

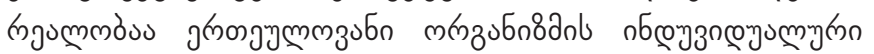
उбmòngrngò (Glasersfeld, 1995).

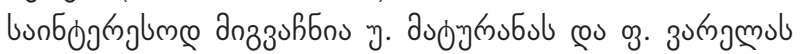

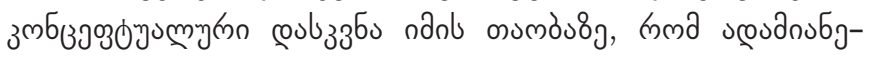

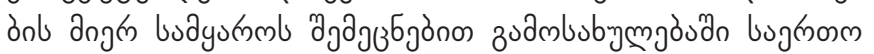

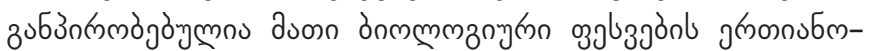

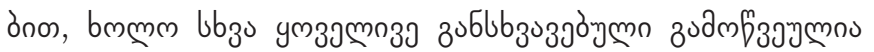

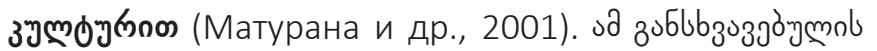

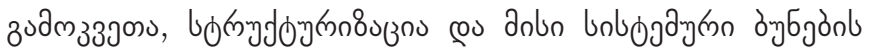

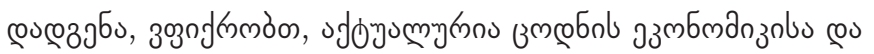

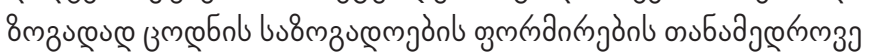

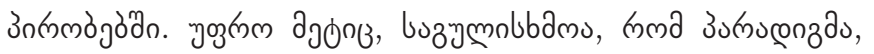

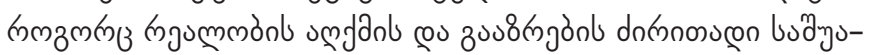

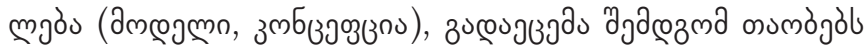

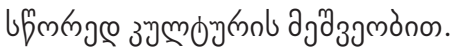

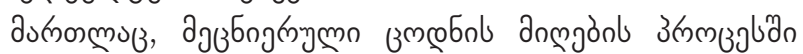

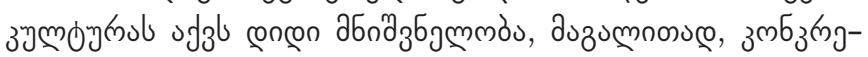

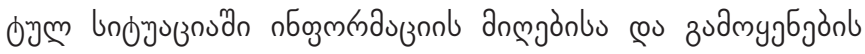

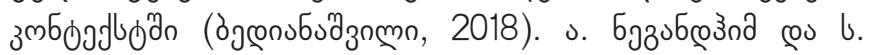

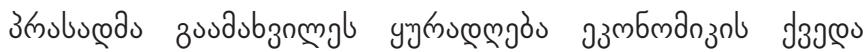

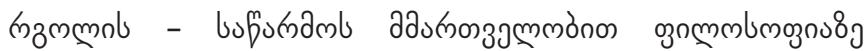

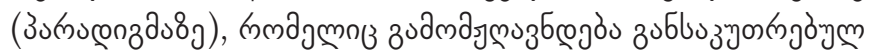

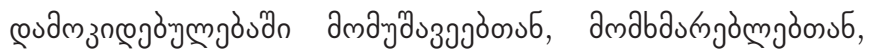

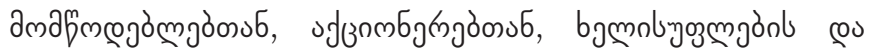

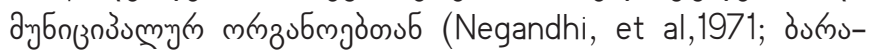

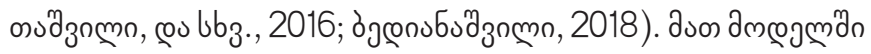

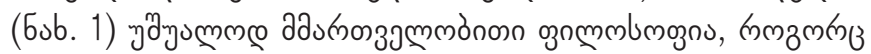

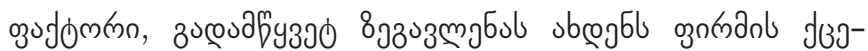

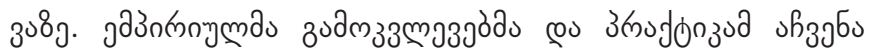

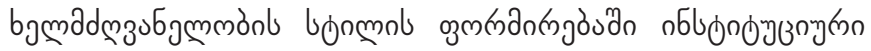

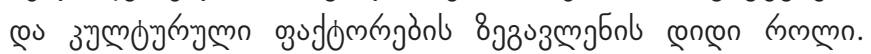

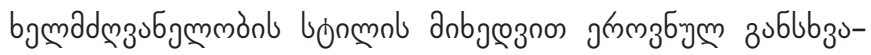

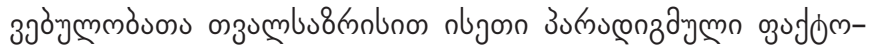

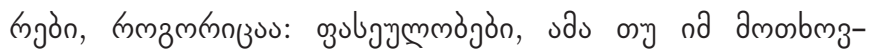

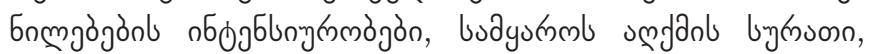
ॠgajoz

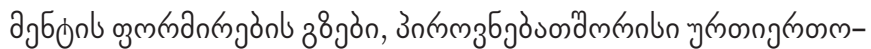

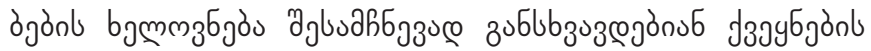

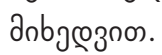

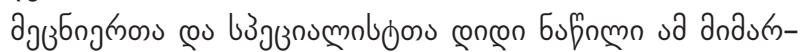

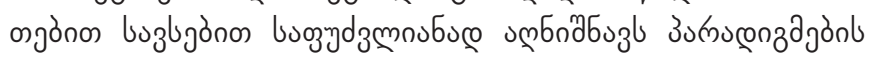

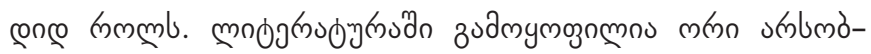

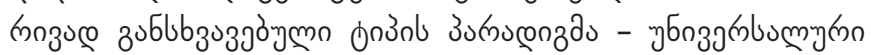

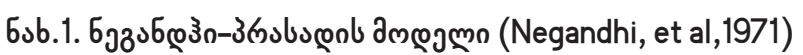

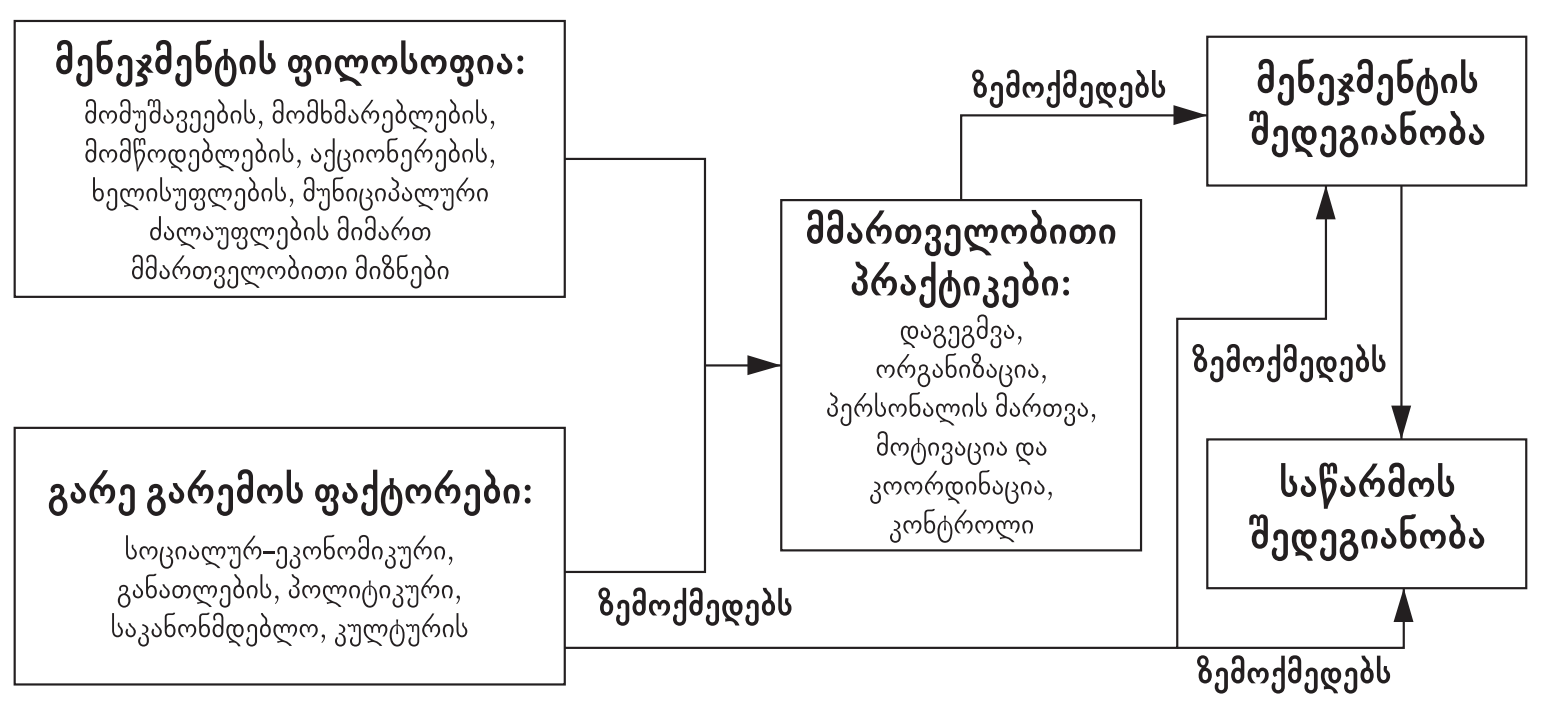




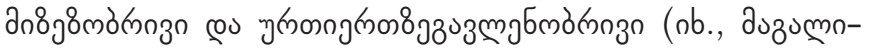
muce, Maruyama, 1974).

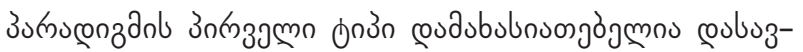

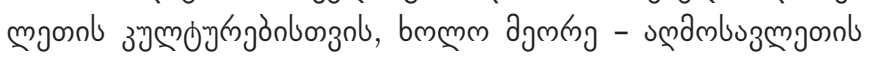

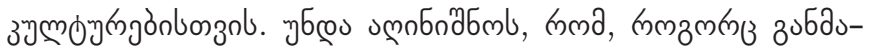

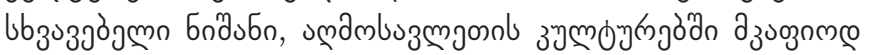

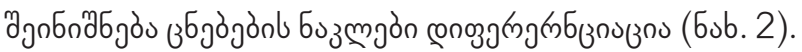

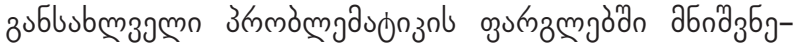

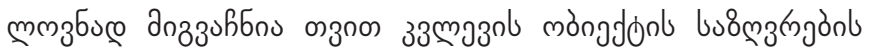

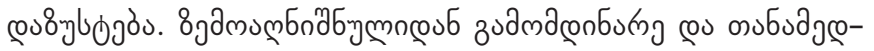

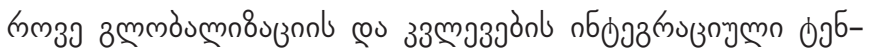

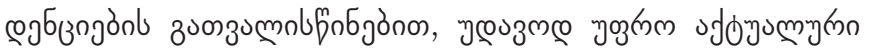

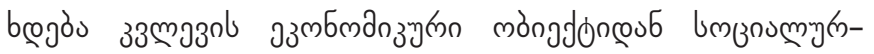

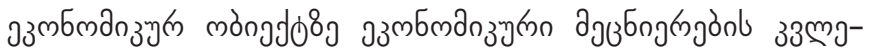

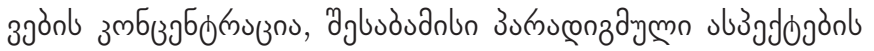

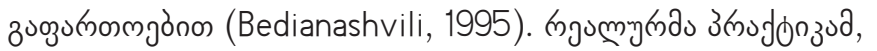

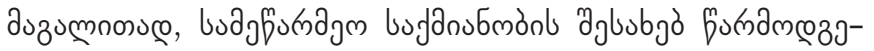

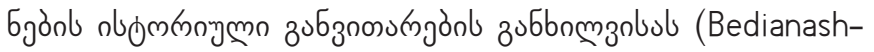

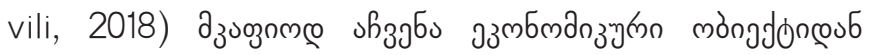

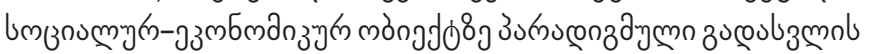

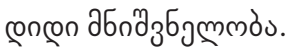

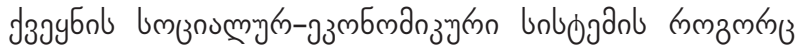

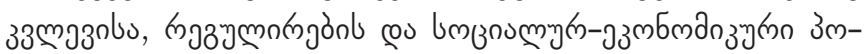

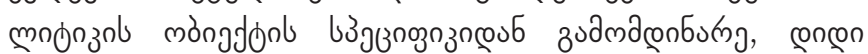

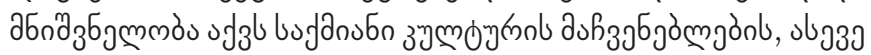

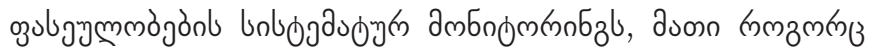

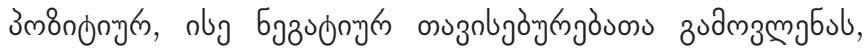

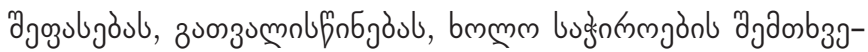

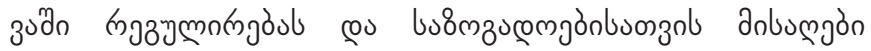

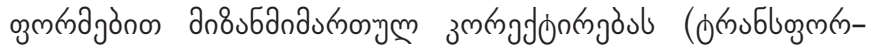

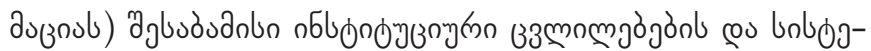

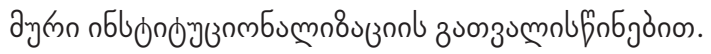

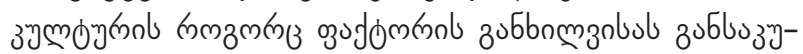

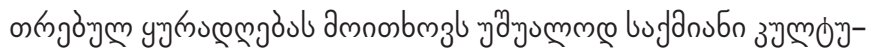

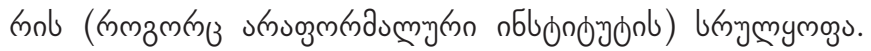

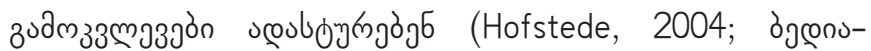

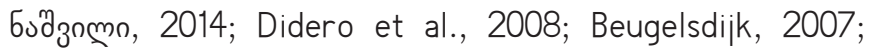
Furman et al., 2002; Geertz, 1973; Herbig and Srholec, 1998; Inglehart and Welzel, 2005; James, 2005; Von Hippel, 2005; Acs, 2006; Barnett, 1953; Beugelsdijk et al., 2014; Brons, 2006; Dickson et al., 2003; Didero et al., 2008; Edler and Fagerberg, 2017; Differences in Inno-

\begin{tabular}{|c|c|c|}
\hline 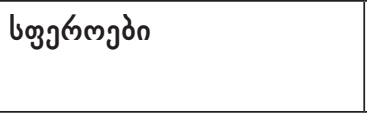 & 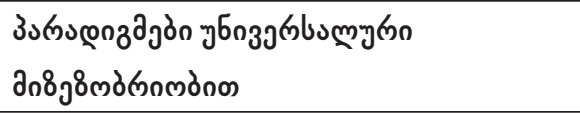 & 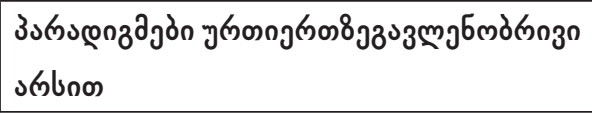 \\
\hline ajzбngmgòs & 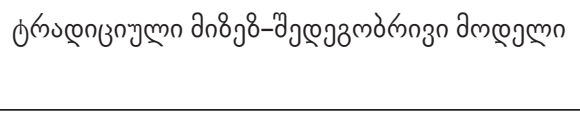 & 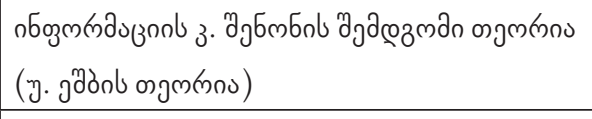 \\
\hline 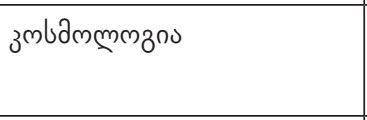 & 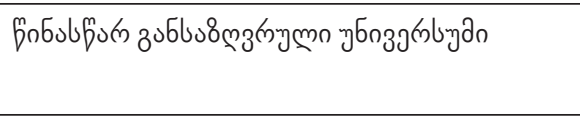 & 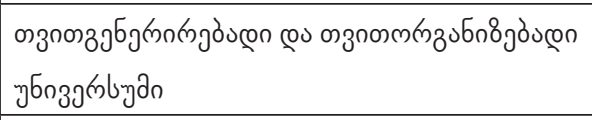 \\
\hline 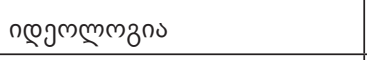 & 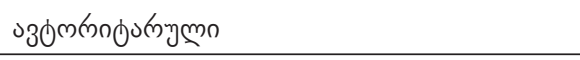 & 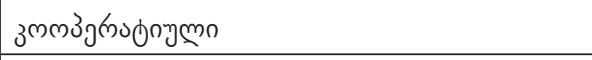 \\
\hline oुnmूmbmoुns & 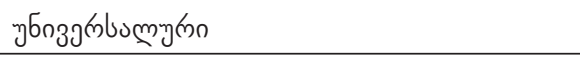 & dbjmynon \\
\hline jon?z० & 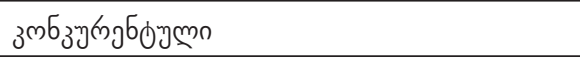 & undznmoynn \\
\hline mamaznos & дmбмmภnвे & 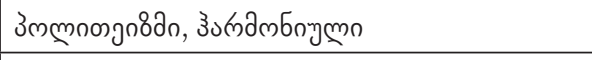 \\
\hline 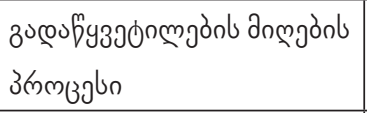 & 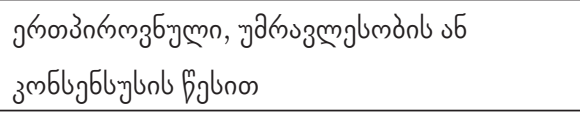 & 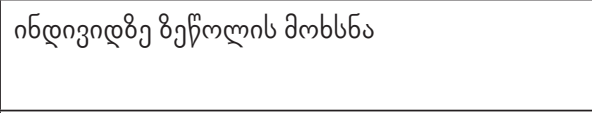 \\
\hline mmznzo & 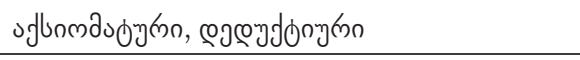 & 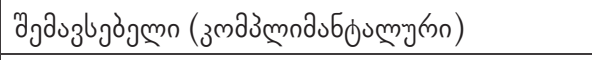 \\
\hline ogjas & 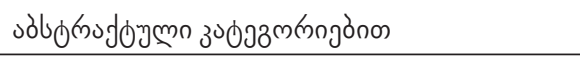 & 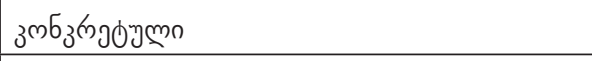 \\
\hline 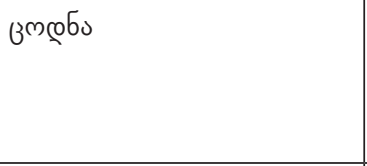 & 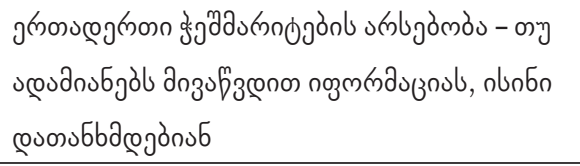 & 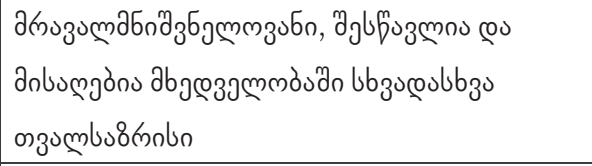 \\
\hline 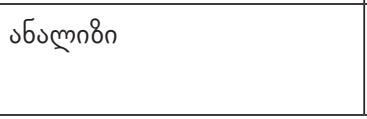 & 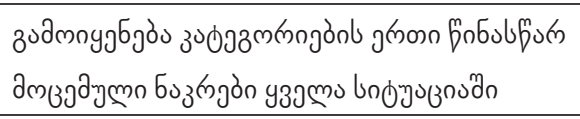 & 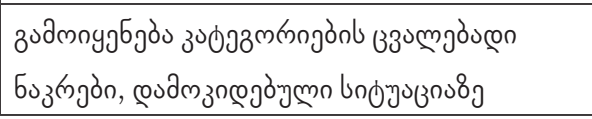 \\
\hline
\end{tabular}

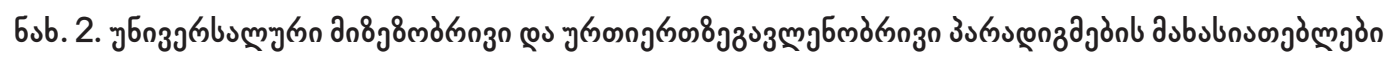




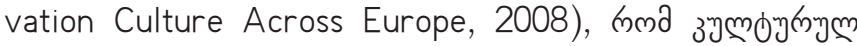

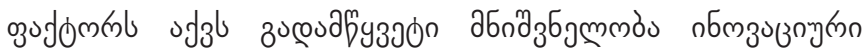

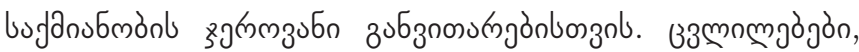

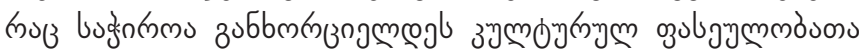

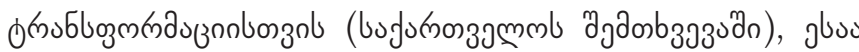

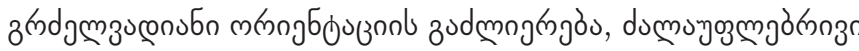

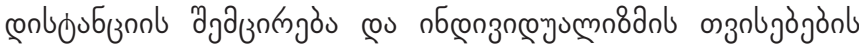

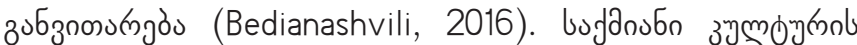
am

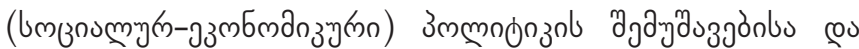

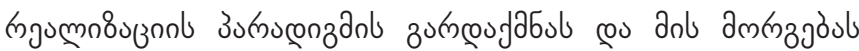

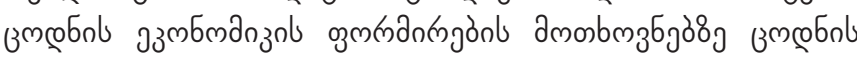

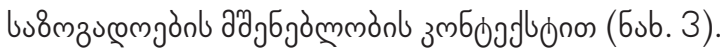

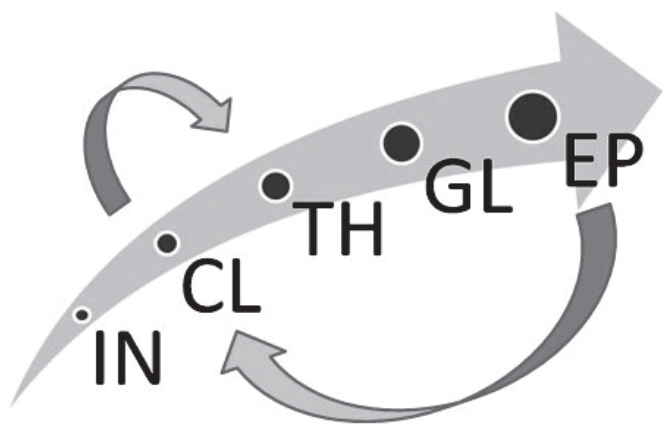

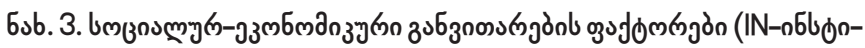

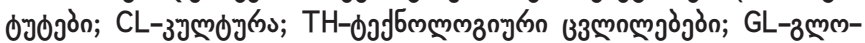

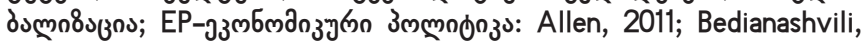
2018)

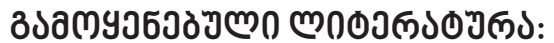

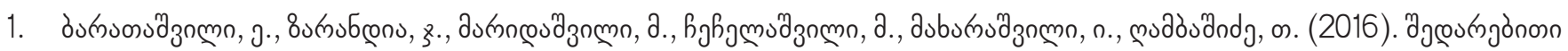

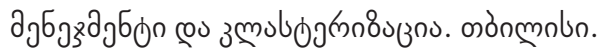

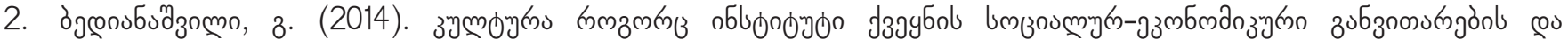

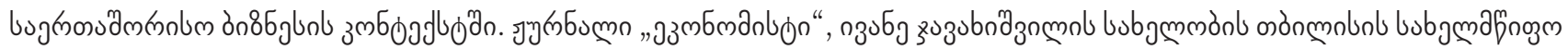

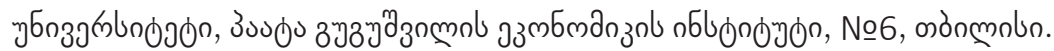

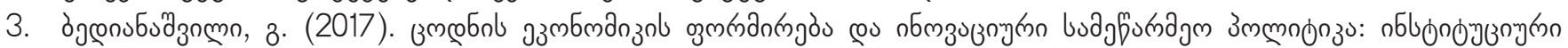

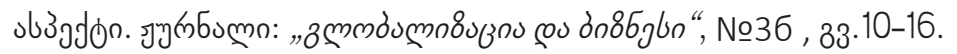

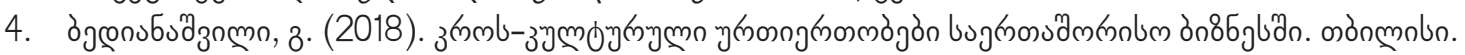

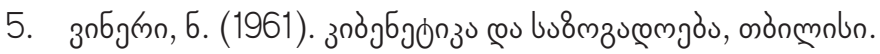

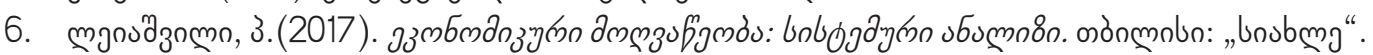

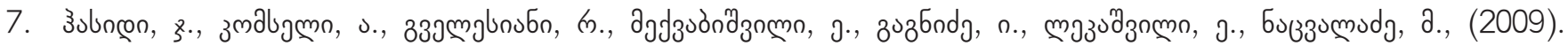

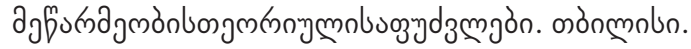

8. Abdelal R., Blyth M., Parsons G. (2009). Constructivist Political Economy.

9. Abdelal, R. (2009). Constructivism as an Approach to International Political Economy. In Handbook of International Political Economy, pp. 57-71.

10. Acs, Z. J., Audretsch, D. B., Braunerhjelm, P. and Carlsson, B., (2005). The Knowledge Spillover Theory of Entrepreneurship, Discussion Papers on Entrepreneurship, Growth and Public Policy, Group Entrepreneurship, Growth and Public Policy, Jena, Germany: Max Planck Institute of Economics.

11. Acs, Z. J., Szerb, L., Lloyd, A., (2018). Global Enterpreneurship Index. Washington, GEDI.

12. Acs, Z.J., (2006). How is Entrepreneurship Good for Economic Growth?, Innovations: Technology, Governance, Globalization 1(1), pp. 97-107.

13. Àsgeirsdòttir, B., (2006). OECD work on knowledge and the knowledge economy. In Brian, K., and Dominique, F. (Eds.), Advancing knowledge and the knowledge Economy. Cambridge: The MIT press, pp.17-23.

14. Augier, M., and Teece, D.J. (2007). Perspectives on research and development. Organizing and Managing innovation in a (Global) knowledge-basedeconomy. In Ichijo, K., and Nonaka, I. (Eds.), Knowledge creation and management. New Challenges for managers. Oxford: Oxford University Press, pp. 198-212.

15. Barnett, H.G. (1953). Innovation: The basis of cultural change. New York: Mc Graw Hill.

16. Baumol W. J. (1990). Enterpreneurship: Productive, Unproductive and Destructive // Journal of Political Economy. Vol. 98. №5. Pt.1, pp. 893-920.

17. Bedianashvili, G. (1995). State, Power Structure and Socio-Economic Reform of Society. Tbilisi, Mecniereba.

18. Bedianashvili, G. (2018). Knowledge Economy, Entrepreneurial Activity and Culture Factor in Modern Conditions of Globalization: Challenges for Georgia. Journal: Globalization and Business, no 5, pp.32-37.. 
19. Beugelsdijk, S. (2007). 'Entrepreneurial culture, regional innovativeness and economic growth', Journal of Evolutionary Economics, 17(1): pp.187-210.

20. Beugelsdijk, S., Slangen, A., Maseland, R., \& Onrust, M. (2014). The impact of home-host cultural distance on foreign affiliate sales: The moderating role of cultural variation within host countries. Journal of Business Research, 67(8), pp.1638-1646.

21. Brons, L. (2006). Indirect measurement of regional culture in the Netherlands, Tijdschrift voor Economische en Sociale Geografie, 97(5): pp.547-566.

22. Constructivism in Theory Knowledge (2008). M., RAS Institute of Philosophy (In Russian).

23. Dahlstrand, Å. L. and Stevenson, L., (2010). Innovative Entrepreneurship Policy: Linking Innovation and Entrepreneurship in a European Context. Annals of Innovation \& Entrepreneurship, vol.1. Retrieved 08. 09. 2012, from http://www.innovationandentrepreneurship.net/index.php/aie/article/view/5602/html_34.

24. Dickson, M. W., Den Hartog, D. N., \& Mitchelson, J. K. (2003). Research on leadership in a cross-cultural context: Making progress, and raising new questions. The leadership quarterly, 14(6), pp.729-768.

25. Didero, M., Gareis, K., Marques, P., \& Ratzke, M. (2008). Differences in innovation culture across Europe. Transformative Use of ICT in EU Regions. Transform, Germany.

26. Didero, M., Gareis, K., Marques, P., Ratzke, M. et al. (2008). Differences in Innovation Culture across Europe, Discussion paper, TRANSFORM project report, URL: http://www.transformeu.org/publications/documents/Differences in Innovation Culture.pdf (retrieved 10. 7. 2008).

27. Differences in Innovation Culture Across Europe (2008).

28. Edler J.,Fagerberg, J. (2017).-Innovation policy: what, why, and how. Oxford Review of Economic Policy. Volume 33, Number 1, pp. pp.2-23.

29. Erqomaishvili G., (2016). Small Entrepreneurship Supporting Policy in Georgia. World Academy of Science, Engineering and Technology International Journal of Economics and Management Engineering Vol: 10, No: 5, pp.1409-1412.

30. European Innovation Scoreboard 2008. Comparative Analysis of Innovation Performance (2009), Pro Inno Europe paper No 10, http://wwwproinno-europe.eu/publications (retrieved, June 2010).

31. European Union EU.(2012). The Innovation Union Scoreboard. Research and Innovation Union scoreboard, Retrieved 07. 20. 2012, from http://www.proinno-europe.eu/metrics

32. Fagerberg, J. and Srholec, M., (2008). National Innovation Systems, Capabilities and Economic Development, Research Policy 37/9, pp.1417-1435.

33. Fagerberg, J., Feldman, M., Shorlec, M., (2011). Technological Dynamics and Social Capability: Comparing U.S. States and European Nations, CIRCLE Paper no 11/2011, Lund University.

34. Foerster H. (2003). Understanding understanding: essays on cybernetics and cognition.

35. Foerster, H. (1995). The Cybernetics of Cybernetics (2nd edition).

36. Foray, D., (2006). The economics of knowledge. Cambridge: The MIT Press.

37. Furman, J. L., Porter, M. E., Stern, S. (2002), TheDeterminants of National Innovative Capacity. Research Policy, 31 (6): 899 933. doi:10.1016/ S0048-7333 (01)00152-4

38. Geertz, C. (1973). The Interpretation of Cultures. New York, Basic Books.

39. Glanville, R. (2008).Second Order Cybernetics. Systems Science and Cybernetics.

40. Glanville, R. (2011). Introduction: A conference doing the cybernetics of cybernetics. Kybernetes, 40(7/8): 952-963.

41. Glasersfeld, E., (1995). Radical Constructivism: A Way of knowing and learning.

42. Gvelesiani R., (2015). Intrinsic Contradictions of Entrepreneurship Development and Self-development. World Academy of Science, Engineering and Technology International Journal of Economics and Management Engineering Vol: 9, No: 3, pp. $1007-1010$.

43. Hadad, S., (2017). Knowledge Economy: Characteristics and Dimensions. Journal: Management Dynamics in the Knowledge Economy Vol.5, no.2, pp.203-225.

44. Hofstede, G et al. (2004) 'Culture's role in entrepreneurship: self-employment out of dissatisfaction', in: Brown, T and Ulijn, J. (eds.) 'Innovation, Entrepreneurship and Culture. The interaction between Technology, Progress and Economic Growth', Cheltenham, Massachusetts: Edward Elgar Publishing, pp. 162-203.

45. Hofstede, G. (2011). Dimensionalizing Cultures: The Hofstede Model in Context. Online Readings in Psychology and Culture, 2(1). https://doi.org/10.9707/2307-0919.1014.

46. http://ppesydney.net/what-is-constructivism-for/ 
47. https://www.researchgate.net/publication/265158222_Constructivist_Political_Economy

48. Inglehart, R. and Welzel, C. (2005) 'Modernization, Cultural Change, and Democracy: The Human Development Sequence', Cambridge, MA, et al.: Cambridge University Press.

49. Konings M. (2015).What is Constructivism For?- Progress in Political Economy (PPE).

50. Kuhn T. (1962). The Structure of Scientific Revolutions.

51. Lakatos I. (1970). Falsification and the Methodology of Scientific Research Programmes.

52. Leiashvily P. (2017). Economic Activity: System Analysis. Tbilisi: Siaxle (In Georgian).

53. Lopez-Leyva, S., Mungaray-Moctezuma A-B., (2017). Knowledge-Based Economy as a Foundation for the Economic Development of Countries. Journal: Management Dynamics in the Knowledge Economy Vol.5 no.4, pp.481-501.

54. Maruyama M. Paradigmatology and Its Application to Cross Disciplinary, Cross Professional and Cross cultural Communication. Dialectica 28 No 3-4.1974.P.135-196.

55. Maturana, H., Varela, F. (1998). The Tree of Knowledge (revised edition). Shambhala Press, Boston.

56. Negandhi A. R., PrasadS.B. (1971). Comparative Management. N. Y.

57. Papava V. (2017). Retroeconomics - Moving from Dying to Brisk Economy. Journal of Reviews on Global Economics, 6, 455462. http://www.lifescienceglobal.com/home/cart?view=product\&id=2567

58. Papava V. (2018). Catching up and Falling Behind: the Experience of the Post-Communist Countries of the European Union and Georgia. GFSIS Expert Opinion, No. 70, Tbilisi. GFSIS. http://papava.info/publications/Papava_Catching-Up-orFalling-Behind_Eng.pdf

59. Papava V., (2016). Technological Backwardness- Global Reality and Expected Challenges for the World's Economy. No. 70. Tbilisi, GFSIS, 2016 (Geo).

60. Papava V., (2017). Retroeconomics - a Theory of the Technological Backwardness of Economy. Nowe ścieżki rozwoju w Europie Środkowej i Nowe ścieżki rozwoju At Nałęczów, Poland, 2017. - pp.33-37.

61. Papava, V. (2016). Technological Backwardness - Global Reality and Expected Challenges for the World's Economy. GFSIS Expert Opinion, No. 70, Tbilisi: GFSIS.

62. Papava V., (1994). The Role of the State in the Modern Economic System. Problems of Economic Transition, Vol. 37 , No. 5.

63. Papava V., Khaduri N. (1997). On the Shadow Political Economy of the Post-Communist Transformation. An Institutional Analysis. Problems of Economic Transition, Vol. 40, No. 6.

64. Petrenko V. (2010). The Paradigm of Constructivism in Humanitarian Sciences. Methodology and History of Psychology. 2010. V. 5. Issue 3, pp. 5-12.

65. Popkov V. (2016). Constructivism and Multiple Economic Theories.Questions of Social Theory. V.8, Issue 1-2 (In Russian).

66. Popper K. (1980). The Logic of Scientific Discovery.

67. Rooka, D., Salvatorip, A., Moylandb, J., Rosab, P., (2017). Innovation Patterns: Upgrading Sectorial Classification for the Fourth Industrial Revolution. Kensho Technologies.

68. Sepashvili E., (2016). Globalized World Economy, Innovations and National Policies for Economic Growth. Governing Business Systems. Theories and Challenges for Systems Thinking in Practice. BSLAB, p.174-176. http://bslab-symposium.net/ Vilnius.2016/BSLab-Vilnius2016-e-book_of_Abstracts.pdf

69. Skrodzka, I. (2016). Knowledge-based economy in the European Union - Crosscountry analysis. Statistics in Transition New Series, 17(2), pp.281-294.

70. Smagina M. (2007). Social Constructivistic Paradigm in Social Knowledge as Alternative of Traditional Methodology. Journal of Sociology and Social Anthropology. V. X, № 2, pp. 73-84.

71. Sundać, D., Krmpotić, I.F. (2011). Knowledge economy factors and the development of knowledge-based economy. Croatia Economic Survey, 13(1), pp.105-141.

72. Tocan, M.C. (2012). Knowledge based economy assessment. Knowledge Management. Retrieved from http://www.scientificpapers.org/knowledgemanagement/knowledge-based-economy-assessment/.

73. Von Hippel, E. (2005). 'Democratizing innovation. Cambridge. MA: MIT Press.

74. White, D.S., Gunasekaran, A., and Ariguzo, G. (2012). The structural components of a knowledge-based economy. International Journal of Business Innovation and Research, 7(4), pp.504-518.

75. Wiener N., (1948). Cybernetics: or Control and Communication in the Animal and the Machine, MIT Press, Cambridge.

76. Алле М., (2004). Основные направления моей работы. М., Мысль.

77. Бир С.. (1963). Кибернетика и управление производством. М., Наука.

78. БлаугМ. (2008). 100 великих экономистовпосле Кейнса. - СПб.:Экономическаяшкола. 
79. ВебленТ.(2007). Теория делового предприятия. М.,Дело.

80. ДрукерП.(2007). Бизнеси инновации. М., «Вильямс».

81. Кобринский Н. , Майминас Е., Смирнов А., (1982). Экономическая кибернетика. М., Экономика.

82. Ланге О. (1968). Введение в экономическую кибернетику. М., Прогресс.

83. Лепский, В. (2015). Экономическая кибернетика саморазвивающихся сред (кибернетика третьего порядка).

84. Макклелланд Д. (2007). Мотивация человека.СПб.,Питер..

85. Матурана, У. (1996). Биология познания. Язык и интеллект. М., с. 95-142.

86. Матурана, У., Варела, Ф. (2001). Древо познания. М.

87. Папава В. (1992). «О некоторых проблемах экономической науки на современном этапе». Вопросы экономики, № 1.

88. Саймон Г. (1995). Теория принятия решений в экономической теории и науке о поведении. СПб., Экономика.

89. Светуньков,М., (2010). Предпринимательство иинновации. Ульяновск., УлГТУ.

90. ШумпетерЙ.А. (2007). Теорияэкономического развития. Капитализм, социализми демократия. М.,Эксмо. 


\section{CULTURE AS A FACTOR OF KNOWLEDGE ECONOMICS WITH PARADIGMATIC CHANGES IN SYSTEMIC INSTITUTIONAL CONTEXT}

GIVI BEDIANASHVILI

https://doi.org/10.35945/gb.2018.06.007

Doctor of Economic Sciences, Professor,

European University,

Academician of the Academy of Economic Sciences of Georgia, Georgia

g.bedianashvili@eu.edu.ge

\section{KEYWORDS: FORMATION OF KNOWLEDGE ECONOMY, CULTURE AS A FACTOR,}

PARADIGMATIC ASPECTS OF ECONOMIC SCIENCE

\section{SUMMARY}

In modern conditions it is important to develop a systemic and institutional concept of culture as a form of knowledge economy. In order to present a systemic concept of knowledge and culture in the formation of knowledge economy, I think the new meaning of paradigmatic and conceptual aspects of economic science is of particular importance. In addition, the systematic representation of the cognition process is particularly important. As shown by the practice of recent years, the main problems in the form of knowledge technology are solved, which will facilitate the improvement of the "cognitive" function of economic science and enhance its practical benefits.

The modern stage of globalization is characterized by a number of specific peculiarities of post-dosystem development, which, in our view, is important to the development of knowledge-based economy (knowledge economy). It is

noteworthy that globalization stems from adding global dimensional dimensions to the knowledge economy, such as resource-technological, informative, communicative and institutional structures. In addition, we think it is necessary to understand the knowledge of the knowledge economy. This makes it possible to develop culture with knowledge as a factor of forming a knowledge economy.

According to the Postindustural Theory, the main resource of the post-denser economy is information and knowledge, the main type of manufacturing activity is the high level of automation of the production, the science of technologies used primarily from the main technologies, and various services are the most common types of economic activity. In industrial society, the field of agriculture, industry - Industry, Postindustry- determinants are already theoretical knowledge with universities, as a place of active and generating knowledge. 